\title{
A note on deficit analysis in dependency models involving Coxian claim amounts
}

\author{
David Landriault*, Wing Yan Lee*, Gordon E. Willmot* and Jae-Kyung Woo ${ }^{\dagger}$
}

October 13, 2012

\begin{abstract}
In this paper, we consider a fairly large class of dependent Sparre Andersen risk models where the claim sizes belong to the class of Coxian distributions. We analyze the Gerber-Shiu discounted penalty function when the penalty function depends on the deficit at ruin. We show that the system of equations needed to solve for this quantity is surprisingly simple. Various applications of this result are also considered.
\end{abstract}

Keywords: dependent Sparre Andersen risk model; Coxian-type claim sizes; time to ruin; deficit at ruin; Lundberg's generalized equation; Lagrange polynomials; Vandemonde matrix; Cauchy matrix.

Acknowledgment: We would like to thank Professor Elias Shiu and an anonymous referee for helpful suggestions. Support for David Landriault and Gordon E. Willmot from grants from the Natural Sciences and Engineering Research Council of Canada is gratefully acknowledged. Support from the Munich Reinsurance Company is also gratefully acknowledged by Gordon E. Willmot as is support from a start-up fund provided by the Faculty of Science and the Department of Statistics and Actuarial Science at the University of Hong Kong by Jae-Kyung Woo.

\footnotetext{
${ }^{*}$ Department of Statistics and Actuarial Science, University of Waterloo, 200 University Avenue West, Waterloo, Canada

${ }^{\dagger}$ Department of Statistics and Actuarial Science, University of Hong Kong, Pokfulam, Hong Kong
} 


\section{Introduction}

In this paper, we model the insurer's surplus process $\left\{U_{t}, t \geq 0\right\}$ by $U_{t}=u+c t-S_{t}$ where $u(u \geq 0)$ is the initial surplus level, $c(c>0)$ is the incoming premium rate per unit time, and $\left\{S_{t}, t \geq 0\right\}$ is a compound renewal process defined as

$$
S_{t}= \begin{cases}\sum_{i=1}^{N_{t}} Y_{i}, & N_{t}>0 \\ 0, & N_{t}=0 .\end{cases}
$$

The claim number process $\left\{N_{t}, t \geq 0\right\}$ is defined through the sequence of interclaim times $\left\{V_{i}\right\}_{i=1}^{\infty}$ which are a collection of independent and identically distributed (iid) random variable (rv)'s with marginal probability density function (pdf) $k$, cumulative distribution function (cdf) $K(t)=1-$ $\bar{K}(t)$, and Laplace transform (LT) $\widetilde{k}(s)=\int_{0}^{\infty} e^{-s t} k(t) d t$. Associated with these claim instants are the claim sizes $\left\{Y_{i}\right\}_{i=1}^{\infty}$, also a sequence of iid rv's with marginal pdf $p, \operatorname{cdf} P(y)=1-\bar{P}(y)$, and LT $\widetilde{p}(s)=\int_{0}^{\infty} e^{-s y} p(y) d y$. We shall assume throughout that $\left\{U_{t}, t \geq 0\right\}$ is a dependent Sparre Andersen risk process, implying that $Y_{i}$ may depend on $V_{i}$, but the pairs $\left\{\left(V_{i}, Y_{i}\right)\right\}_{i=1}^{\infty}$ are iid. We therefore denote the joint pdf of an arbitrary pair $\left(V_{i}, Y_{i}\right)$ at $(t, y)$ by $p(y \mid t) k(t)$. Recently, various authors have examined dependency models of this nature. Interested readers are referred to Albrecher and Teugels (2006), Badescu et al. (2009), Boudreault et al. (2006), Chadjiconstantinidis and Vrontos (2012), Cheung et al. (2010), Cossette et al. (2008), Cheung (2011) and Willmot and Woo (2012) to name a few.

Here we consider a fairly large class of dependent Sparre Andersen risk models by modelling the joint pdf of $\left(V_{i}, Y_{i}\right)$ as

$$
p(y \mid t) k(t)=\sum_{i=1}^{m} \sum_{j=1}^{n_{i}} g_{i j}(t) e_{\beta_{i}, j}(y), \quad t, y \geq 0,
$$

where $e_{\beta, j}(y)$ is the Erlang density

$$
e_{\beta, j}(y)=\frac{\beta(\beta y)^{j-1} e^{-\beta y}}{(j-1) !}, \quad y>0 .
$$

Obviously, the marginal pdfs of $V_{i}$ and $Y_{i}$ are respectively given by

$$
k(t)=\sum_{i=1}^{m} \sum_{j=1}^{n_{i}} g_{i j}(t), \quad t \geq 0,
$$

and

$$
p(y)=\sum_{i=1}^{m} \sum_{j=1}^{n_{i}} \eta_{i j} e_{\beta_{i}, j}(y), \quad y \geq 0,
$$

with $\eta_{i j}=\int_{0}^{\infty} g_{i j}(t) d t$. We remark that the claim sizes have marginal density (3) which is a Coxian- $n$ pdf with $n=\sum_{i=1}^{m} n_{i}$.

The class of joint pdfs (1) is very large, and the results we obtain in this paper thus hold quite generally. A few notable special cases are first discussed. When $g_{i j}(t)=g(t)$ for all $i$ and $j$, the resulting risk model is the ordinary Sparre Andersen model with interclaim time pdf $n \cdot g()$ and 
claim size pdf (3). Also, when $g_{i j}$ is either a non-negative or non-positive function for all $i$ and $j$, the joint pdf can be rewritten as

$$
p(y \mid t) k(t)=\sum_{i=1}^{m} \sum_{j=1}^{n_{i}} \eta_{i j} k_{i j}(t) e_{\beta_{i}, j}(y), \quad t, y \geq 0,
$$

where $k_{i j}(t)=g_{i j}(t) / \eta_{i j}$ is a pdf. In this case, the joint density function of $\left(V_{i}, Y_{i}\right)$ is a combination/mixture of joint pdfs with independent components.

Note that it is not necessary for $g_{i j}$ to be either a non-negative or non-positive function for (1) to be a valid joint pdf. Therefore, in what follows we do not assume $k_{i j}$ (whenever $\eta_{i j} \neq 0$ ) to be a probability density function (pdf). An example of this is the dependent renewal risk model where the dependence between $V$ and $Y$ is introduced through the Farlie-Gumbel-Morgenstern (FGM) copula (see, e.g., Nelsen (1994)). That is, we assume that

$$
\operatorname{Pr}(V \leq t, Y \leq y)=C_{F G M}(K(t), P(y)),
$$

for $t, y \geq 0$ where

$$
C_{F G M}(u, v)=u v+\theta u v(1-u)(1-v),
$$

with $|\theta| \leq 1$. Routine calculations lead to the following joint pdf of $\left(V_{i}, Y_{i}\right)$ :

$$
p(y \mid t) k(t)=p(y) k(t)[1+\theta\{1-2 P(y)\}\{1-2 K(t)\}], \quad t, y \geq 0 .
$$

When $Y_{i}$ is an exponential $\mathrm{rv}$ with mean $1 / \beta$, (5) simplifies to

$$
p(y \mid t) k(t)=k(t)[1-\theta\{1-2 K(t)\}] e_{\beta, 1}(y)+\theta k(t)\{1-2 K(t)\} e_{2 \beta, 1}(y),
$$

which is of the form (1) with $m=2, n_{1}=n_{2}=1, \beta_{1}=\beta, \beta_{2}=2 \beta, g_{11}(t)=k(t)[1-\theta\{1-2 K(t)\}]$, and $g_{21}(t)=\theta k(t)\{1-2 K(t)\}$. Note that $g_{21}$ is neither a non-negative nor a non-positive function. More generally, it is not difficult to show that when

$$
\operatorname{Pr}(V \leq t, Y \leq y)=C(K(t), P(y)),
$$

where $C(u, v)$ is a polynomial copula in $v$ (see Nelsen (2006, Chapter 3)) and $P(y)$ is the cdf associated with a finite combination/mixture of Erlangs with possibly different scale parameters, then the joint pdf of $(V, Y)$ belongs to the class of joint pdfs (1). Examples of polynomial copulas (i.e. copulas with polynomial sections in one or more variables) are the FGM copula, the generalized FGM copula (see, e.g. Cossette et al. (2008)), and the very versatile Bernstein copula (see, e.g., Sancetta and Satchell (2004)).

Landriault and Willmot (2008) demonstrated that, for the ordinary Sparre Andersen model with interclaim time pdf $g$ and claim size pdf (3), the Gerber-Shiu function corresponding to a particular class of penalty functions is completely characterized through the solution of a linear system of equations. In the present paper, a slightly simpler penalty function is considered which only involves the deficit at ruin, but the more general dependent Sparre Andersen model is considered. In Section 2 it is shown that in this situation the Gerber-Shiu function is still characterized through a linear system of equations, and this linear system is substantially simpler than that obtained by Landriault and Willmot (2008) with the more general penalty function. In fact, the resulting linear system is reminiscient of that involving the use of a block Vandermonde type of matrix.

In Section 3 we consider evaluation of the Gerber-Shiu function for particular choices of the penalty function. This allows for evaluation of various ruin-related quantities, including the distribution of the deficit at ruin. Finally, in Section 4 special cases of the dependence model (1) are considered which allow for direct solution of the linear system of equations even with a general penalty function. 


\section{$2 \quad$ A simplified linear system}

Let $T$ be the time to ruin for the surplus process $\left\{U_{t}, t \geq 0\right\}$, i.e. $T=\inf \left\{t \geq 0: U_{t}<0\right\}$ with $T=\infty$ if $U_{t} \geq 0$ for all $t \geq 0$. To characterize the joint discounted density $h_{\delta, 12}(x, y \mid u)$ of the surplus prior to ruin $(x)$ and the deficit at ruin $(y)$, we first examine the nature of the joint distribution of the time to ruin $T$, the surplus prior to ruin $U_{T^{-}}$, the deficit at ruin $\left|U_{T}\right|$, and the surplus after the second last claim before ruin $R_{N_{T}-1}=u+\sum_{i=1}^{N_{T}-1}\left(c V_{i}-Y_{i}\right)$. If ruin occurs on the first claim, Cheung et al. (2010) showed that the joint (defective) density of the surplus prior to ruin $(x)$ and the deficit at ruin $(y)$ is

$$
h_{1}(x, y \mid u)=\frac{1}{c} k\left(\frac{x-u}{c}\right) p\left(x+y \mid \frac{x-u}{c}\right),
$$

and in this case the time to ruin is $T=\frac{x-u}{c}$ and $R_{N_{T}-1}=u$. If ruin occurs on claims subsequent to the first, we denote by $h_{2}(t, x, y, v \mid u)$ the joint (defective) density of the time to ruin $(t)$, the surplus prior to ruin $(x)$, the deficit at ruin $(y)$, and the surplus after the second last claim before ruin $(v)$. Using simple probabilistic arguments, we have

$$
\begin{aligned}
h_{2}(t, x, y, v \mid u) & =\left\{\int_{0}^{\infty} h_{2}(t, x, y, v \mid u) d y\right\} \frac{h_{1}(x, y \mid v)}{\int_{0}^{\infty} h_{1}(x, y \mid v) d y} \\
& =\left\{\int_{0}^{\infty} h_{2}(t, x, y, v \mid u) d y\right\} \frac{\frac{1}{c} k\left(\frac{x-v}{c}\right) p\left(x+y \mid \frac{x-v}{c}\right)}{\int_{0}^{\infty} \frac{1}{c} k\left(\frac{x-u}{c}\right) p\left(x+y \mid \frac{x-u}{c}\right) d y} .
\end{aligned}
$$

One can argue the validity of $(7)$ as follows: the joint density of $\left(T, U_{T^{-}},\left|U_{T}\right|, R_{N_{T}-1}\right)$ at $(t, x, y, v)$ is the product of the joint density of $\left(T, U_{T^{-}}, R_{N_{T}-1}\right)$ at $(t, x, v)$ and the (conditional) density of the deficit at ruin of $y$ given that ruin occurs on a last ascent of the surplus process from level $v$ to $x$ (without claim in the interim) which is given by $h_{1}(x, y \mid v) / \int_{0}^{\infty} h_{1}(x, y \mid v) d y$.

From Cheung et al. (2010), the discounted density $h_{\delta, 12}(x, y \mid u)$ is given by

$$
h_{\delta, 12}(x, y \mid u)=e^{-\delta\left(\frac{x-u}{c}\right)} h_{1}(x, y \mid u)+\int_{0}^{\infty} e^{-\delta t} \int_{0}^{x} h_{2}(t, x, y, v \mid u) d v d t .
$$

Substituting (1) into (6), (7) and subsequently (8), one finds

$$
h_{\delta, 12}(x, y \mid u)=\sum_{i=1}^{m} \sum_{j=1}^{n_{i}} \eta_{\delta, i j}(x \mid u) e_{\beta_{i}, j}(x+y),
$$

where

$$
\begin{aligned}
\eta_{\delta, i j}(x \mid u)= & \frac{1}{c} e^{-\frac{\delta}{c}(x-u)} g_{i j}\left(\frac{x-u}{c}\right) \\
& +\int_{0}^{\infty} e^{-\delta t} \int_{0}^{x}\left\{\int_{0}^{\infty} h_{2}(t, x, y, v \mid u) d y\right\} \frac{g_{i j}\left(\frac{x-v}{c}\right)}{\sum_{i=1}^{m} \sum_{j=1}^{n_{i}} \bar{e}_{\beta_{i}, j}(x) g_{i j}\left(\frac{x-v}{c}\right)} d v d t
\end{aligned}
$$

with $\bar{e}_{\beta, j}(x)=\int_{x}^{\infty} e_{\beta, j}(y) d y$. We can also find an alternative expression for the discounted joint density $h_{\delta, 12}(x, y \mid u)$ similar to (9). From Lemma 1 in Cheung (2011), we know that the discounted pdf $h_{2, \delta}(x, y, v \mid u)=\int_{0}^{\infty} e^{-\delta t} h_{2}(t, x, y, v \mid u) d t$ factors as

$$
h_{2, \delta}(x, y, v \mid u)=\tau_{\delta}(u, v) h_{1, \delta}(x, y \mid v), \quad x>v>0 ; y>0
$$


where $h_{1, \delta}(x, y \mid u)=e^{-\delta\left(\frac{x-u}{c}\right)} h_{1}(x, y \mid u)$ and some function $\tau_{\delta}(u, v)$ defined in Eq (2.7) of Cheung (2011). Then substitution of (11) into (8) yields a similar form of (9). We remark that this $\tau_{\delta}(0, v)$ when $\delta=0$ is essentially same as the density of the renewal function associated with the ascending ladder height of the current random walk as studied by Pitts and Politis (2007) (also e.g. Tang and Wei (2010), and Zhang et al. (2011)).

Although (10) is complicated, its exact form is not relevant in what follows, as the representation (9) for the joint discounted density is what is of interest. Nevertheless, it is interesting to note that if $g_{i j}(t)=g(t)$ for all $i$ and $j$, then (10) simplifies to

$$
\eta_{\delta, i j}(x \mid u)=\frac{h_{\delta, 1}(x \mid u)}{\bar{P}(x)}
$$

where

$$
h_{\delta, 1}(x \mid u)=\frac{1}{c} e^{-\frac{\delta}{c}(x-u)} g\left(\frac{x-u}{c}\right) \bar{P}(x)+\int_{0}^{\infty} e^{-\delta t} \int_{0}^{x} \int_{0}^{\infty} h_{2}(t, x, y, v \mid u) d y d v d t,
$$

is the discounted density of the surplus prior to ruin at $x$.

We remark that the Erlang density $e_{\beta, j}$ allows the factorization

$$
e_{\beta, j}(x+y)=\frac{1}{\beta} \sum_{k=1}^{j} e_{\beta, j+1-k}(x) e_{\beta, k}(y),
$$

(see, e.g., Willmot (2007, Eq. 3.26)) which implies that (9) becomes

$$
h_{\delta, 12}(x, y \mid u)=\sum_{i=1}^{m} \sum_{k=1}^{n_{i}} \xi_{\delta, i k}(x \mid u) e_{\beta_{i}, k}(y),
$$

with

$$
\xi_{\delta, i k}(x \mid u)=\frac{1}{\beta_{i}} \sum_{j=k}^{n_{i}} \eta_{\delta, i j}(x \mid u) e_{\beta_{i}, j+1-k}(x) .
$$

Using (13), the proper discounted density of the descending ladder height is given by

$$
\begin{aligned}
b_{\delta}(y) & =\frac{1}{\phi_{\delta}} \int_{0}^{\infty} h_{\delta, 12}(x, y \mid 0) d x \\
& =\sum_{i=1}^{m} \sum_{k=1}^{n_{i}} \xi_{\delta, i k} e_{\beta_{i}, k}(y)
\end{aligned}
$$

where

$$
\xi_{\delta, i k}=\frac{1}{\phi_{\delta}} \int_{0}^{\infty} \xi_{\delta, i k}(x \mid 0) d x
$$

and

$$
\phi_{\delta}=\int_{0}^{\infty} \int_{0}^{\infty} h_{\delta, 12}(x, y \mid 0) d x d y .
$$

We consider evaluation of the Gerber-Shiu function

$$
m_{\delta}(u)=E\left[e^{-\delta T} w\left(\left|U_{T}\right|\right) 1(T<\infty) \mid U_{0}=u\right],
$$


where the penalty function $w$ is assumed to be a function of the deficit at ruin only (see, e.g., Landriault and Willmot (2008) for the analysis of a more general penalty function in a similar context to the present one, but the analysis is significantly more complex in this case). From Cheung et al. (2010), $m_{\delta}(u)$ satisfies the defective renewal equation

$$
m_{\delta}(u)=\phi_{\delta} \int_{0}^{u} m_{\delta}(u-y) b_{\delta}(y) d y+v_{\delta}(u),
$$

with

$$
v_{\delta}(u)=\phi_{\delta} \int_{0}^{\infty} w(y) b_{\delta}(u+y) d y
$$

Using (14), this may be expressed again as

$$
\begin{aligned}
v_{\delta}(u) & =\phi_{\delta} \int_{0}^{\infty} w(y)\left\{\sum_{i=1}^{m} \sum_{k=1}^{n_{i}} \xi_{\delta, i k} e_{\beta_{i}, k}(u+y)\right\} d y \\
& =\phi_{\delta} \sum_{i=1}^{m} \sum_{k=1}^{n_{i}} \xi_{\delta, i k} \int_{0}^{\infty} w(y)\left\{\frac{1}{\beta_{i}} \sum_{j=1}^{k} e_{\beta_{i}, j}(u) e_{\beta_{i}, k+1-j}(y)\right\} d y \\
& =\phi_{\delta} \sum_{i=1}^{m} \sum_{k=1}^{n_{i}} \sum_{j=1}^{k} e_{\beta_{i}, j}(u) \frac{\xi_{\delta, i k}}{\beta_{i}} \int_{0}^{\infty} w(y) e_{\beta_{i}, k+1-j}(y) d y \\
& =\phi_{\delta} \sum_{i=1}^{m} \sum_{j=1}^{n_{i}} e_{\beta_{i}, j}(u) \sum_{k=j}^{n_{i}} \frac{\xi_{\delta, i k}}{\beta_{i}} E\left[w\left(E_{i, k+1-j}\right)\right]
\end{aligned}
$$

where $E_{i, j}$ has pdf $e_{\beta_{i}, j}$, and thus

$$
E\left[w\left(E_{i, j}\right)\right]=\int_{0}^{\infty} w(y) e_{\beta_{i}, j}(y) d y .
$$

That is,

$$
v_{\delta}(u)=\sum_{i=1}^{m} \sum_{j=1}^{n_{i}} \xi_{\delta, i j}^{*} e_{\beta_{i, j}}(u),
$$

where

$$
\xi_{\delta, i j}^{*}=\phi_{\delta} \sum_{k=j}^{n_{i}} \frac{\xi_{\delta, i k}}{\beta_{i}} E\left[w\left(E_{i, k+1-j}\right)\right] .
$$

In what follows we denote the LT of an arbitrary function $a$ by $\widetilde{a}(s)=\int_{0}^{\infty} e^{-s x} a(x) d x$ (whenever it exists). It follows from (14) and (18) that the defective renewal equation (15) may be expressed in Laplace transform form as

$$
\begin{aligned}
\widetilde{m}_{\delta}(s) & =\frac{\widetilde{v}_{\delta}(s)}{1-\phi_{\delta} \widetilde{b}_{\delta}(s)} \\
& =\frac{\sum_{i=1}^{m} \sum_{j=1}^{n_{i}} \xi_{\delta, i j}^{*}\left(\frac{\beta_{i}}{\beta_{i}+s}\right)^{j}}{1-\phi_{\delta} \sum_{i=1}^{m} \sum_{j=1}^{n_{i}} \xi_{\delta, i j}\left(\frac{\beta_{i}}{\beta_{i}+s}\right)^{j}} .
\end{aligned}
$$


Thus,

$$
\widetilde{m}_{\delta}(s)=\frac{\left\{\prod_{k=1}^{m}\left(\beta_{k}+s\right)^{n_{k}}\right\} \sum_{i=1}^{m} \sum_{j=1}^{n_{i}} \xi_{\delta, i j}^{*}\left(\frac{\beta_{i}}{\beta_{i}+s}\right)^{j}}{\left\{\prod_{k=1}^{m}\left(\beta_{k}+s\right)^{n_{k}}\right\}\left\{1-\phi_{\delta} \sum_{i=1}^{m} \sum_{j=1}^{n_{i}} \xi_{\delta, i j}\left(\frac{\beta_{i}}{\beta_{i}+s}\right)^{j}\right\}} .
$$

It is clear that the numerator in (20) is a polynomial of degree $n-1$ (or less) in $s$. Similarly, the denominator is a polynomial of degree $n$. Thus, the denominator has $n$ roots in the complex plane, say $-R_{1, \delta},-R_{2, \delta}, \ldots,-R_{n, \delta}$. Assuming that these $n$ roots are distinct, it follows that (20) may be expressed, after a partial fraction expansion, as

$$
\widetilde{m}_{\delta}(s)=\sum_{k=1}^{n} \frac{C_{k, \delta}}{s+R_{k, \delta}}
$$

where $C_{1, \delta}, C_{2, \delta}, \ldots, C_{n, \delta}$ are constants. Then inversion of (21) yields

$$
m_{\delta}(u)=\sum_{k=1}^{n} C_{k, \delta} e^{-R_{k, \delta} u}, \quad u \geq 0 .
$$

In what follows, we further assume that the $R_{k, \delta}$ 's are also distinct from the $\beta_{i}$ 's. This is well known to be true in special cases of the model involving mixtures (e.g. Gerber and Shiu (1998, p.69)).

The next step is to show that for the Gerber-Shiu function (22) resulting from the joint density (1), the $R_{k, \delta}$ 's may be obtained as negative roots of Lundberg's generalized equation, and the $C_{k, \delta}$ 's satisfy a surprisingly simple linear system of equations. Before giving the main result, it is useful to first make a few minor technical remarks about the $R_{k, \delta}$ 's and the $C_{k, \delta}$ 's.

First, we note that the $n$ zeros $-R_{1, \delta},-R_{2, \delta}, \ldots,-R_{n, \delta}$ of the denominator in (21) can be arbitrarily ordered. Thus, if at least one of the $C_{k, \delta}$ 's is 0 , all $C_{k, \delta}$ 's must be 0 and $m_{\delta}(u)=0$ for all $u \geq 0$. In what follows, we assume that $m_{\delta}(u) \neq 0$. Furthermore, as commonly assumed, we consider Gerber-Shiu functions $m_{\delta}(u)$ that vanish at infinity which implies that the zeros $-R_{1, \delta},-R_{2, \delta}, \ldots,-R_{n, \delta}$ must all have a negative real part. In Theorem 1 , these zeros $-R_{1, \delta},-R_{2, \delta}, \ldots,-R_{n, \delta}$ are shown to be solutions of Lundberg's generalized equation

$$
E\left[e^{-\delta V} e^{-s(Y-c V)}\right]=1 .
$$

That is, $R_{k, \delta}$ satisfies

$$
\sum_{i=1}^{m} \sum_{j=1}^{n_{i}}\left(\frac{\beta_{i}}{\beta_{i}-R_{k, \delta}}\right)^{j} \widetilde{g}_{i j}\left(\delta+c R_{k, \delta}\right)=1 .
$$

Henceforth, we tacitly assume that $\left|\widetilde{g}_{i j}\left(\delta+c R_{k, \delta}\right)\right|<\infty$ for all $i, j, k$, so that Lundberg's generalized equation (24) is well defined. A standard Rouche-type argument is normally used to prove that there are exactly $n$ solutions to (23) with a negative real part. The argument typically proceeds as follows. For $\delta>0$, it is clear that

$$
\left|E\left[e^{-\delta V} e^{-s(Y-c V)}\right]\right| \leq E\left[e^{-\delta V}\right]<1,
$$

for $\operatorname{Re}(s)=0$. For the part of the circle (centered at 0 ) of radius $r$ with a negative real part,

$$
\left|E\left[e^{-\delta V} e^{-s(Y-c V)}\right]\right| \leq \sum_{i=1}^{m} \sum_{j=1}^{n_{i}}\left|\widetilde{g}_{i j}(\delta-c s)\right|\left|\left(\frac{\beta_{i}}{\beta_{i}+s}\right)^{j}\right|,
$$


where $\left|\widetilde{g}_{i j}(\delta-c s)\right|$ is finite for $\operatorname{Re}(s)<0$ if $g_{i j}$ is absolutely integrable. Then, for a sufficiently large $r$

$$
\left|E\left[e^{-\delta V} e^{-s(Y-c V)}\right]\right| \leq \sum_{i=1}^{m} \sum_{j=1}^{n_{i}}\left|\widetilde{g}_{i j}(\delta-c s)\right|\left|\left(\frac{\beta_{i}}{\beta_{i}+s}\right)^{j}\right|<1,
$$

for all $\{s:|s|=r$ and $\operatorname{Re}(s)<0\}$. Given that $\prod_{i=1}^{m}\left(\beta_{i}+s\right)^{n_{i}}=0$ has $n$ solutions with a negative real part, this is also true for $E\left[e^{-\delta V} e^{-s(Y-c V)}\right]=1$. A similar proof leads to an identical conclusion when $\delta=0$ and the security loading is assumed to be positive (i.e. $E\left[c V_{i}-Y_{i}\right]>0$ ).

We now state the main result of this paper.

Theorem 1 Consider the dependent Sparre Andersen risk model with joint density $p(y \mid t) k(t)$ given by (1) and a Gerber-Shiu function $m_{\delta}(u)$ of the form (22) with $\beta_{1}, \beta_{2}, \ldots, \beta_{m}$ and $R_{1, \delta}, R_{2, \delta}, \ldots, R_{n, \delta}$ all distinct. Then for $k=1,2, \ldots, n, R_{k, \delta}$ satisfies Lundberg's generalized equation (24). Furthermore, as long as $\widetilde{g}_{i n_{i}}\left(\delta+c \beta_{i}\right) \neq 0$ for $i=1,2, \ldots, m$, it follows that $C_{1, \delta}, C_{2, \delta}, \ldots, C_{n, \delta}$ satisfy the linear system of equations

$$
\sum_{k=1}^{n} C_{k, \delta}\left(\frac{\beta_{i}}{\beta_{i}-R_{k, \delta}}\right)^{j}=E\left[w\left(E_{i, j}\right)\right],
$$

for $i=1,2, \ldots, m$ and $j=1,2, \ldots, n_{i}$, with $E\left[w\left(E_{i, j}\right)\right]$ given by (17).

Proof: By conditioning on the time and the amount of the first claim, we have

$$
\begin{aligned}
m_{\delta}(u)= & \int_{0}^{\infty} e^{-\delta t} \int_{u+c t}^{\infty} w(y-u-c t) p(y \mid t) k(t) d y d t \\
& +\int_{0}^{\infty} e^{-\delta t} \int_{0}^{u+c t} m_{\delta}(u+c t-y) p(y \mid t) k(t) d y d t .
\end{aligned}
$$

Substitution of (1) and (22) into (26) yields

$$
\begin{aligned}
\sum_{k=1}^{n} C_{k, \delta} e^{-R_{k, \delta} u}= & \int_{0}^{\infty} e^{-\delta t} \int_{u+c t}^{\infty} w(y-u-c t)\left\{\sum_{i=1}^{m} \sum_{h=1}^{n_{i}} g_{i h}(t) e_{\beta_{i}, h}(y)\right\} d y d t \\
& +\int_{0}^{\infty} e^{-\delta t} \int_{0}^{u+c t}\left\{\sum_{k=1}^{n} C_{k, \delta} e^{-R_{k, \delta}(u+c t-y)}\right\}\left\{\sum_{i=1}^{m} \sum_{h=1}^{n_{i}} g_{i h}(t) e_{\beta_{i}, h}(y)\right\} d y d t \\
& =\sum_{i=1}^{m} \sum_{h=1}^{n_{i}} \int_{0}^{\infty} e^{-\delta t}\left\{\int_{0}^{\infty} w(y) e_{\beta_{i}, h}(y+u+c t) d y\right\} g_{i h}(t) d t \\
& +\sum_{i=1}^{m} \sum_{k=1}^{n} C_{k, \delta} \sum_{h=1}^{n_{i}} \int_{0}^{\infty} e^{-\delta t}\left\{\int_{0}^{u+c t} e^{-R_{k, \delta}(u+c t-y)} e_{\beta_{i}, h}(y) d y\right\} g_{i h}(t) d t .
\end{aligned}
$$

We now consider the two inner integrals in this expression. First, using (12),

$$
\begin{aligned}
\int_{0}^{\infty} w(y) e_{\beta_{i}, h}(y+u+c t) d y & =\frac{1}{\beta_{i}} \int_{0}^{\infty} w(y)\left\{\sum_{l=1}^{h} e_{\beta_{i}, h-l+1}(y) e_{\beta_{i}, l}(u+c t)\right\} d y \\
& =\frac{1}{\beta_{i}} \sum_{l=1}^{h}\left\{\int_{0}^{\infty} w(y) e_{\beta_{i}, h-l+1}(y) d y\right\} e_{\beta_{i}, l}(u+c t) \\
& =\frac{1}{\beta_{i}} \sum_{l=1}^{h} E\left[w\left(E_{i, h-l+1}\right)\right] e_{\beta_{i}, l}(u+c t) .
\end{aligned}
$$


Second, one has easily that for $R \neq \beta_{i}$,

$$
\int_{0}^{u} e^{-R(u-y)} e_{\beta_{i}, h}(y) d y=\left(\frac{\beta_{i}}{\beta_{i}-R}\right)^{h} e^{-R u}-\frac{1}{\beta_{i}} \sum_{l=1}^{h}\left(\frac{\beta_{i}}{\beta_{i}-R}\right)^{h-l+1} e_{\beta_{i}, l}(u) .
$$

Thus,

$$
\begin{aligned}
\sum_{k=1}^{n} C_{k, \delta} e^{-R_{k, \delta} u}= & \sum_{i=1}^{m} \sum_{h=1}^{n_{i}} \int_{0}^{\infty} e^{-\delta t}\left\{\frac{1}{\beta_{i}} \sum_{l=1}^{h} E\left[w\left(E_{i, h-l+1}\right)\right] e_{\beta_{i}, l}(u+c t)\right\} g_{i h}(t) d t \\
& +\sum_{i=1}^{m} \sum_{k=1}^{n} C_{k, \delta} \sum_{h=1}^{n_{i}} \int_{0}^{\infty} e^{-\delta t}\left\{\left(\frac{\beta_{i}}{\beta_{i}-R_{k, \delta}}\right)^{h} e^{-R_{k, \delta}(u+c t)}\right\} g_{i h}(t) d t \\
& -\sum_{i=1}^{m} \sum_{k=1}^{n} C_{k, \delta} \sum_{h=1}^{n_{i}} \int_{0}^{\infty} e^{-\delta t}\left\{\frac{1}{\beta_{i}} \sum_{l=1}^{h}\left(\frac{\beta_{i}}{\beta_{i}-R_{k, \delta}}\right)^{h-l+1} e_{\beta_{i}, l}(u+c t)\right\} g_{i h}(t) d t \\
= & \sum_{i=1}^{m} \sum_{h=1}^{n_{i}} \sum_{l=1}^{h} \frac{1}{\beta_{i}} E\left[w_{2}\left(E_{i, h-l+1}\right)\right] \int_{0}^{\infty} e^{-\delta t} e_{\beta_{i}, l}(u+c t) g_{i h}(t) d t \\
& +\sum_{i=1}^{m} \sum_{k=1}^{n} C_{k, \delta} \sum_{j=1}^{n_{i}}\left(\frac{\beta_{i}}{\beta_{i}-R_{k, \delta}}\right)^{j} e^{-R_{k, \delta} u} \int_{0}^{\infty} e^{-\left(\delta+c R_{k, \delta}\right) t} g_{i j}(t) d t \\
& -\sum_{i=1}^{m} \sum_{k=1}^{n} C_{k, \delta} \sum_{h=1}^{n_{i}} \sum_{l=1}^{h} \frac{1}{\beta_{i}}\left(\frac{\beta_{i}}{\beta_{i}-R_{k, \delta}}\right)^{h-l+1} \int_{0}^{\infty} e^{-\delta t} e_{\beta_{i}, l}(u+c t) g_{i h}(t) d t,
\end{aligned}
$$

where the index of summation has been changed from $h$ to $j$ on the second last line. In turn, using (12) again,

$$
\begin{aligned}
\frac{1}{\beta_{i}} \int_{0}^{\infty} e^{-\delta t} e_{\beta_{i}, l}(u+c t) g_{i h}(t) d t & =\frac{1}{\beta_{i}^{2}} \int_{0}^{\infty} e^{-\delta t}\left\{\sum_{j=1}^{l} e_{\beta_{i}, j}(u) e_{\beta_{i}, l-j+1}(c t)\right\} g_{i h}(t) d t \\
& =\frac{1}{\beta_{i}^{2}} \sum_{j=1}^{l} e_{\beta_{i}, j}(u) \int_{0}^{\infty} e^{-\delta t} e_{\beta_{i}, l-j+1}(c t) g_{i h}(t) d t \\
& =\sum_{j=1}^{l} e_{\beta_{i}, j}(u) M_{i, h, l-j+1}^{*}(\delta)
\end{aligned}
$$

where

$$
M_{i, h, l}^{*}(\delta)=\frac{1}{\beta_{i}^{2}} \int_{0}^{\infty} e^{-\delta t} e_{\beta_{i}, l}(c t) g_{i h}(t) d t .
$$


Therefore,

$$
\begin{aligned}
\sum_{k=1}^{n} C_{k, \delta}\left\{1-\sum_{i=1}^{m} \sum_{j=1}^{n_{i}}\left(\frac{\beta_{i}}{\beta_{i}-R_{k, \delta}}\right)^{j} \widetilde{g}_{i j}\left(\delta+c R_{k, \delta}\right)\right\} e^{-R_{k, \delta} u} \\
=\sum_{i=1}^{m} \sum_{h=1}^{n_{i}} \sum_{l=1}^{h} E\left[w\left(E_{i, h-l+1}\right)\right] \sum_{j=1}^{l} e_{\beta_{i}, j}(u) M_{i, h, l-j+1}^{*}(\delta) \\
-\sum_{i=1}^{m} \sum_{k=1}^{n} C_{k, \delta} \sum_{h=1}^{n_{i}} \sum_{l=1}^{h}\left(\frac{\beta_{i}}{\beta_{i}-R_{k, \delta}}\right)^{h-l+1} \sum_{j=1}^{l} e_{\beta_{i}, j}(u) M_{i, h, l-j+1}^{*}(\delta) \\
=\sum_{i=1}^{m} \sum_{h=1}^{n_{i}} \sum_{j=1}^{h} e_{\beta_{i}, j}(u) \sum_{l=j}^{h} E\left[w\left(E_{i, h-l+1}\right)\right] M_{i, h, l-j+1}^{*}(\delta) \\
-\sum_{i=1}^{m} \sum_{k=1}^{n} C_{k, \delta} \sum_{h=1}^{n_{i}} \sum_{j=1}^{h} e_{\beta_{i}, j}(u) \sum_{l=j}^{h}\left(\frac{\beta_{i}}{\beta_{i}-R_{k, \delta}}\right)^{h-l+1} M_{i, h, l-j+1}^{*}(\delta) \\
=\sum_{i=1}^{m} \sum_{j=1}^{n_{i}} e_{\beta_{i}, j}(u) \sum_{h=j}^{n_{i}} \sum_{l=j}^{h} E\left[w_{2}\left(E_{i, h-l+1}\right)\right] M_{i, h, l-j+1}^{*}(\delta) \\
-\sum_{i=1}^{m} \sum_{k=1}^{n} C_{k, \delta} \sum_{j=1}^{n_{i}} e_{\beta_{i}, j}(u) \sum_{h=1}^{n_{i}} \sum_{l=j}^{h}\left(\frac{\beta_{i}}{\beta_{i}-R_{k, \delta}}\right)^{h-l+1} M_{i, h, l-j+1}^{*}(\delta) .
\end{aligned}
$$

Hence,

$$
\begin{aligned}
& \sum_{k=1}^{n} C_{k, \delta}\left\{1-\sum_{i=1}^{m} \sum_{j=1}^{n_{i}}\left(\frac{\beta_{i}}{\beta_{i}-R_{k, \delta}}\right)^{j} \widetilde{g}_{i j}\left(\delta+c R_{k, \delta}\right)\right\} e^{-R_{k, \delta} u} \\
& =\sum_{i=1}^{m} \sum_{j=1}^{n_{i}} e_{\beta_{i, j}}(u)\left\{\sum_{h=j}^{n_{i}} \sum_{l=j}^{h} M_{i, h, l-j+1}^{*}(\delta)\left(E\left[w\left(E_{i, h-l+1}\right)\right]-\sum_{k=1}^{n} C_{k, \delta}\left(\frac{\beta_{i}}{\beta_{i}-R_{k, \delta}}\right)^{h-l+1}\right)\right\} .
\end{aligned}
$$

Now, (28) is true for all $u \geq 0$, and thus the coefficients of $e^{-R_{k, \delta} u}$ for $k=1,2, \ldots, n$ and $e_{\beta_{i}, j}(u)$ for $i=1,2, \ldots, m$ and $j=1,2, \ldots, n_{i}$ must all be zero. Therefore, equating the coefficient of $e^{-R_{k, \delta} u}$ to zero yields $(24)$. For $e_{\beta_{i}, j}(u)$, it follows that

$$
\sum_{h=j}^{n_{i}} \sum_{l=j}^{h} M_{i, h, l-j+1}^{*}(\delta)\left\{E\left[w\left(E_{i, h-l+1}\right)\right]-\sum_{k=1}^{n} C_{k, \delta}\left(\frac{\beta_{i}}{\beta_{i}-R_{k, \delta}}\right)^{h-l+1}\right\}=0,
$$

for $i=1,2, \ldots, m$ and $j=1,2, \ldots, n_{i}$. To simplify (29), define (for notational convenience)

$$
f_{i, j}(\delta)=E\left[w\left(E_{i, j+1}\right)\right]-\sum_{k=1}^{n} C_{k, \delta}\left(\frac{\beta_{i}}{\beta_{i}-R_{k, \delta}}\right)^{j+1},
$$


and thus the left side of (29) becomes

$$
\begin{aligned}
\sum_{h=j}^{n_{i}} \sum_{l=j}^{h} M_{i, h, l-j+1}^{*}(\delta) f_{i, h-l}(\delta) & =\sum_{l=j}^{n_{i}} \sum_{h=l}^{n_{i}} M_{i, h, l-j+1}^{*}(\delta) f_{i, h-l}(\delta) \\
& =\sum_{l=j}^{n_{i}} \sum_{h=0}^{n_{i}-l} M_{i, h+l, l-j+1}^{*}(\delta) f_{i, h}(\delta) \\
& =\sum_{h=0}^{n_{i}-j} f_{i, h}(\delta) \sum_{l=j}^{n_{i}-h} M_{i, h+l, l-j+1}^{*}(\delta) \\
& =\sum_{h=0}^{n_{i}-j} f_{i, h}(\delta) \sum_{l=0}^{n_{i}-(h+j)} M_{i, h+j+l, l+1}^{*}(\delta) .
\end{aligned}
$$

Thus, with

$$
M_{i, h}(\delta)=\sum_{l=0}^{n_{i}-h} M_{i, h+l, l+1}^{*}(\delta),
$$

(29) may be re-expressed as

$$
\sum_{h=0}^{n_{i}-j} f_{i, h}(\delta) M_{i, j+h}(\delta)=0,
$$

again for $i=1,2, \ldots, m$ and $j=1,2, \ldots, n_{i}$. For $j=n_{i},(31)$ becomes

$$
f_{i, 0}(\delta) M_{i, n_{i}}(\delta)=0
$$

From (27) and (30), we know that

$$
M_{i, n_{i}}(\delta)=M_{i, n_{i}, 1}^{*}(\delta)=\frac{\widetilde{g}_{i n_{i}}\left(\delta+c \beta_{i}\right)}{\beta_{i}},
$$

which is assumed to be non-zero. Therefore, $f_{i, 0}(\delta)$ must be 0 for $(32)$ to hold, that is

$$
\sum_{k=1}^{n} C_{k, \delta}\left(\frac{\beta_{i}}{\beta_{i}-R_{k, \delta}}\right)=E\left[w\left(E_{i, 1}\right)\right] .
$$

By an inductive argument, let us now assume that

$$
f_{i, j}(\delta)=0, \quad j=0,1, \ldots, k-1,
$$

and prove that $f_{i, k}(\delta)=0$. Using (31) at $j=n_{i}-k$ (note that (31) holds for $j=1, \ldots, n_{i}$ which implies that $\left.k \in\left\{0,1, \ldots, n_{i}-1\right\}\right)$, it follows that

$$
\sum_{h=0}^{k} f_{i, h}(\delta) M_{i, n_{i}-k+h}(\delta)=0 .
$$

Under (34), it is clear that (35) holds if

$$
f_{i, k}(\delta) M_{i, n_{i}}(\delta)=0
$$


or equivalently

$$
f_{i, k}(\delta)=0 \text {. }
$$

It follows that $f_{i, k}(\delta)=0$ for $k=0,1, \ldots, n_{i}-1$ which yields $(25)$.

As noted in the context of the copula example in Section 1, it may well be the case that $g_{i j}(t)$ is neither a positive or a negative function of $t$. It is useful to note however that for mixture or combination models with $g_{i n_{i}}(t)=\eta_{i n_{i}} k_{i n_{i}}(t)$ where $k_{i n_{i}}(t)$ is a pdf for all $i=1,2, \ldots, m$, then the integral condition in the statement of Theorem 1 (namely, $\widetilde{g}_{i n_{i}}\left(\delta+c \beta_{i}\right) \neq 0$ ) is automatically satisfied. This is because $\eta_{i n_{i}}=0$ would then imply that $n_{i}$ may be replaced (without loss of generality) by $n_{i}-1$ in the joint pdf (4) (or alternatively (1)).

\section{Particular penalty functions and the deficit at ruin}

In Section 2, it was shown that the Gerber-Shiu function is characterized by solutions of Lundberg's generalized equation together with a linear system of equations for the associated coefficients. In this section, we employ a different approach to characterize the form of these coefficients. This form is seen to be fairly complicated in general, but simplifies for some special cases of the penalty function which allow for analysis of the deficit.

Considering the ladder height pdf (14), it is clear that

$$
\left\{\prod_{i=1}^{m}\left(s+\beta_{i}\right)^{n_{i}}\right\} \widetilde{b}_{\delta}(s)=\left\{\prod_{i=1}^{m}\left(s+\beta_{i}\right)^{n_{i}}\right\} \sum_{i=1}^{m} \sum_{j=1}^{n_{i}} \xi_{\delta, i j}\left(\frac{\beta_{i}}{\beta_{i}+s}\right)^{j}
$$

is a polynomial of degree $n-1$ or less. Thus,

$$
\left\{\prod_{i=1}^{m}\left(s+\beta_{i}\right)^{n_{i}}\right\}\left\{1-\phi_{\delta} \widetilde{b}_{\delta}(s)\right\}
$$

is a polynomial of degree $n$ in $s$ with (leading) coefficient of $s^{n}$ equal to 1 , and from (20), $s=-R_{j, \delta}$ is a solution of the equation $1-\phi_{\delta} \widetilde{b}_{\delta}(s)=0$ for $j=1,2, \ldots, n$. This polynomial therefore equals $\prod_{j=1}^{n}\left(s+R_{j, \delta}\right)$, i.e.

$$
1-\phi_{\delta} \widetilde{b}_{\delta}(s)=\frac{\prod_{j=1}^{n}\left(s+R_{j, \delta}\right)}{\prod_{i=1}^{m}\left(s+\beta_{i}\right)^{n_{i}}} .
$$

As $\widetilde{b}_{\delta}(0)=1,(36)$ implies that

$$
\phi_{\delta}=1-\frac{\prod_{j=1}^{n} R_{j, \delta}}{\prod_{i=1}^{m} \beta_{i}^{n_{i}}}
$$

and in turn

$$
\tilde{b}_{\delta}(s)=\frac{\left\{\prod_{i=1}^{m}\left(s+\beta_{i}\right)^{n_{i}}\right\}-\left\{\prod_{j=1}^{n}\left(s+R_{j, \delta}\right)\right\}}{\phi_{\delta}\left\{\prod_{i=1}^{m}\left(s+\beta_{i}\right)^{n_{i}}\right\}},
$$


with $\phi_{\delta}$ given by (37).

Note that from (14),

$$
\widetilde{b}_{\delta}(s)=\sum_{i=1}^{m} \sum_{j=1}^{n_{i}} \xi_{\delta, i j}\left(\frac{\beta_{i}}{\beta_{i}+s}\right)^{j},
$$

and so from the theory of partial fractions,

$$
\xi_{\delta, i j}=\left.\frac{\beta_{i}^{-j}}{\left(n_{i}-j\right) !}\left\{\frac{d^{n_{i}-j}}{d s^{n_{i}-j}}\left[\left(s+\beta_{i}\right)^{n_{i}} \widetilde{b}_{\delta}(s)\right]\right\}\right|_{s=-\beta_{i}},
$$

with $\widetilde{b}_{\delta}(s)$ given by (38). Of course, (39) is straightforward in principle to evaluate but tedious in general.

Furthermore, for $k=1,2, \ldots, n$, it follows from (36) that

$$
\frac{1-\phi_{\delta} \widetilde{b}_{\delta}(s)}{s+R_{k, \delta}}=\frac{\prod_{j=1, j \neq k}^{n}\left(s+R_{j, \delta}\right)}{\prod_{i=1}^{m}\left(s+\beta_{i}\right)^{n_{i}}},
$$

and in turn,

$$
\lim _{s \rightarrow-R_{k, \delta}} \frac{1-\phi_{\delta} \widetilde{b}_{\delta}(s)}{s+R_{k, \delta}}=\frac{\prod_{j=1, j \neq k}^{n}\left(R_{j, \delta}-R_{k, \delta}\right)}{\prod_{i=1}^{m}\left(\beta_{i}-R_{k, \delta}\right)^{n_{i}}} .
$$

Equations (15) and (21) together imply that

$$
\widetilde{m}_{\delta}(s)=\sum_{k=1}^{n} \frac{C_{k, \delta}}{s+R_{k, \delta}}=\frac{\widetilde{v}_{\delta}(s)}{1-\phi_{\delta} \widetilde{b}_{\delta}(s)},
$$

and so using (40)

$$
\begin{aligned}
C_{k, \delta} & =\lim _{s \rightarrow-R_{k, \delta}}\left(s+R_{k, \delta}\right) \tilde{m}_{\delta}(s) \\
& =\widetilde{v}_{\delta}\left(-R_{k, \delta}\right) \frac{\prod_{i=1}^{m}\left(\beta_{i}-R_{k, \delta}\right)^{n_{i}}}{\prod_{j=1, j \neq k}^{n}\left(R_{j, \delta}-R_{k, \delta}\right)} .
\end{aligned}
$$

An explicit expression for $\widetilde{v}_{\delta}\left(-R_{k, \delta}\right)$ in (41) is available using (18) and (19) by analytic continuation, but (39) is awkward in general. Simplification occurs with some choices of $w(y)$ however. For example, if $w(y)=e^{-z y}$, then from $(16), v_{\delta}(u)=\mathcal{T}_{z}\left\{\phi_{\delta} b_{\delta}(u)\right\}$ where $\mathcal{T}_{z}$ is the Dickson-Hipp operator defined as $\mathcal{T}_{z} f(u)=\int_{0}^{\infty} e^{-z y} f(u+y) d y$ for $\operatorname{Re} z \geq 0$ (see, e.g., Li and Garrido (2004, Section 3)). Thus, when $w(y)=e^{-z y}$,

$$
\widetilde{v}_{\delta}(s)=\frac{\phi_{\delta} \widetilde{b}_{\delta}(z)-\phi_{\delta} \widetilde{b}_{\delta}(s)}{s-z}
$$

and then (42) is easily evaluated with the help of (38). Similarly, when $w(y)=y^{n}$ where $n$ is a positive integer, $\widetilde{v}_{\delta}(s)$ may be expressed in terms of $\widetilde{b}_{\delta}(s)$ using ideas of Lin and Willmot (1999, 
2000), but the details are omitted. When $z=0$ so that $w(y)=1$ for all $y$, then (42) with $z=0$ implies that (41) reduces to

$$
C_{k, \delta}^{*}=\left\{\prod_{i=1}^{m}\left(\frac{\beta_{i}-R_{k, \delta}}{\beta_{i}}\right)^{n_{i}}\right\}\left\{\prod_{j=1, j \neq k}^{n}\left(\frac{R_{j, \delta}}{R_{j, \delta}-R_{k, \delta}}\right)\right\} .
$$

Note that the Laplace transform of the time to ruin is obtained when $w(y)=1$ which implies that

$$
\bar{G}_{\delta}(u)=\sum_{k=1}^{n} C_{k, \delta}^{*} e^{-R_{k, \delta} u}, \quad u \geq 0
$$

with $C_{k, \delta}^{*}$ given by (43). The ruin probability $\psi(u)=\bar{G}_{0}(u)$ is the further special case $\delta=0$. Also, the compound geometric density $g_{\delta}(u)=-\bar{G}_{\delta}^{\prime}(u)$ is given by

$$
g_{\delta}(u)=\sum_{k=1}^{n} C_{k, \delta}^{*} R_{k, \delta} e^{-R_{k, \delta} u}, \quad u>0 .
$$

Next, we focus on the marginal (discounted) distribution of the deficit at ruin $\left|U_{T}\right|$. For the analysis, we consider $m_{\delta}(u)$ with $w(y)=e^{-z y}$ which satisfies the defective renewal equation (15). With $w(y)=e^{-z y}$, we have

$$
\begin{aligned}
m_{\delta}(u) & \equiv E\left[e^{-\delta T-z\left|U_{T}\right|} I_{\{T<\infty\}} \mid U_{0}=u\right] \\
& =\int_{0}^{\infty} e^{-z y} h_{\delta, 2}(y \mid u) d y
\end{aligned}
$$

where

$$
h_{\delta, 2}(y \mid u)=\int_{0}^{\infty} h_{\delta, 12}(x, y \mid u) d x
$$

is the marginal discounted density of the deficit at ruin. Using, e.g., Theorem 9.1.1 of Willmot and Lin (2000), the solution to (15) may be expressed as

$$
m_{\delta}(u)=v_{\delta}(u)+\frac{1}{1-\phi_{\delta}} \int_{0}^{u} v_{\delta}(t) g_{\delta}(u-t) d t,
$$

where $g_{\delta}(u)$ is given by $(45)$ and

$$
v_{\delta}(u)=\phi_{\delta} \int_{0}^{\infty} e^{-z y} b_{\delta}(u+y) d y
$$

Thus, (48) becomes

$$
\begin{aligned}
m_{\delta}(u) & =\phi_{\delta} \int_{0}^{\infty} e^{-z y} b_{\delta}(u+y) d y+\frac{\phi_{\delta}}{1-\phi_{\delta}} \int_{0}^{u} \int_{0}^{\infty} e^{-z y} b_{\delta}(t+y) g_{\delta}(u-t) d y d t \\
& =\phi_{\delta} \int_{0}^{\infty} e^{-z y}\left\{b_{\delta}(u+y)+\frac{1}{1-\phi_{\delta}} \int_{0}^{u} b_{\delta}(t+y) g_{\delta}(u-t) d t\right\} d y,
\end{aligned}
$$

which yields

$$
h_{\delta, 2}(y \mid u)=\frac{\phi_{\delta}}{1-\phi_{\delta}}\left\{\left(1-\phi_{\delta}\right) b_{\delta}(u+y)+\int_{0}^{u} b_{\delta}(t+y) g_{\delta}(u-t) d t\right\} .
$$


Let

$$
h_{\delta, 2, u}(y)=\frac{h_{\delta, 2}(y \mid u)}{\bar{G}_{\delta}(u)},
$$

be the proper discounted density of the deficit at ruin which can be expressed as

$$
h_{\delta, 2, u}(y)=\frac{\phi_{\delta}\left(1-\phi_{\delta}\right) b_{\delta}(u+y)+\phi_{\delta} \int_{0}^{u} b_{\delta}(t+y) g_{\delta}(u-t) d t}{\left(1-\phi_{\delta}\right) \bar{G}_{\delta}(u)} .
$$

Using (14) and (12), one has

$$
\begin{aligned}
b_{\delta}(x+y) & =\sum_{i=1}^{m} \sum_{h=1}^{n_{i}} \xi_{\delta, i h} e_{\beta_{i}, h}(x+y) \\
& =\sum_{i=1}^{m} \sum_{h=1}^{n_{i}} \frac{\xi_{\delta, i h}}{\beta_{i}} \sum_{j=1}^{h} e_{\beta_{i}, j}(y) e_{\beta_{i}, h-j+1}(x) \\
& =\sum_{i=1}^{m} \sum_{j=1}^{n_{i}} e_{\beta_{i}, j}(y)\left\{\sum_{h=j}^{n_{i}} \frac{\xi_{\delta, i h}}{\beta_{i}} e_{\beta_{i}, h-j+1}(x)\right\} .
\end{aligned}
$$

Thus, (49) becomes

$$
\begin{aligned}
h_{\delta, 2, u}(y)= & \frac{\phi_{\delta}}{\bar{G}_{\delta}(u)} \sum_{i=1}^{m} \sum_{j=1}^{n_{i}} e_{\beta_{i}, j}(y)\left\{\sum_{h=j}^{n_{i}} \frac{\xi_{\delta, i h}}{\beta_{i}} e_{\beta_{i, h-j+1}}(u)\right\} \\
& +\frac{\phi_{\delta}}{\left(1-\phi_{\delta}\right) \bar{G}_{\delta}(u)} \int_{0}^{u}\left\{\sum_{i=1}^{m} \sum_{j=1}^{n_{i}} e_{\beta_{i}, j}(y) \sum_{h=j}^{n_{i}} \frac{\xi_{\delta, i h}}{\beta_{i}} e_{\beta_{i}, h-j+1}(t)\right\} g_{\delta}(u-t) d t \\
= & \frac{\phi_{\delta}}{\bar{G}_{\delta}(u)} \sum_{i=1}^{m} \sum_{j=1}^{n_{i}} e_{\beta_{i}, j}(y)\left\{\sum_{h=j}^{n_{i}} \frac{\xi_{\delta, i h}}{\beta_{i}} e_{\beta_{i}, h-j+1}(u)\right\} \\
& +\frac{\phi_{\delta}}{\left(1-\phi_{\delta}\right) \bar{G}_{\delta}(u)} \sum_{i=1}^{m} \sum_{j=1}^{n_{i}} e_{\beta_{i}, j}(y)\left\{\sum_{h=j}^{n_{i}} \frac{\xi_{\delta, i h}}{\beta_{i}} \int_{0}^{u} e_{\beta_{i}, h-j+1}(t) g_{\delta}(u-t) d t\right\}
\end{aligned}
$$

That is,

$$
h_{\delta, 2, u}(y)=\sum_{i=1}^{m} \sum_{j=1}^{n_{i}} \bar{\xi}_{\delta, i j}(u) e_{\beta_{i}, j}(y)
$$

where

$$
\bar{\xi}_{\delta, i j}(u)=\frac{\phi_{\delta}}{\left(1-\phi_{\delta}\right) \bar{G}_{\delta}(u)} \sum_{h=j}^{n_{i}} \frac{\xi_{\delta, i h}}{\beta_{i}}\left\{\left(1-\phi_{\delta}\right) e_{\beta_{i}, h-j+1}(u)+\int_{0}^{u} e_{\beta_{i}, h-j+1}(t) g_{\delta}(u-t) d t\right\},
$$


and thus $h_{\delta, 2, u}(y)$ is again of the same Coxian- $n$ form. Also, as (44) holds and from (45),

$$
\begin{aligned}
& \int_{0}^{u} e_{\beta_{i}, h-j+1}(t) g_{\delta}(u-t) d t \\
& =\int_{0}^{u} e_{\beta_{i}, h-j+1}(t)\left\{\sum_{k=1}^{n} C_{k, \delta}^{*} R_{k, \delta} e^{-R_{k, \delta}(u-t)}\right\} d t \\
& =\sum_{k=1}^{n} C_{k, \delta}^{*} R_{k, \delta} \int_{0}^{u} e^{-R_{k, \delta}(u-t)} e_{\beta_{i}, h-j+1}(t) d t \\
& =\sum_{k=1}^{n} C_{k, \delta}^{*} R_{k, \delta}\left\{\left(\frac{\beta_{i}}{\beta_{i}-R_{k, \delta}}\right)^{h-j+1} e^{-R_{k, \delta} u}-\frac{1}{\beta_{i}} \sum_{\ell=1}^{h-j+1}\left(\frac{\beta_{i}}{\beta_{i}-R_{k, \delta}}\right)^{h-j-\ell+2} e_{\beta_{i}, \ell}(u)\right\},
\end{aligned}
$$

it follows that (51) and hence (50) are straightforward to evaluate.

\section{Special cases of the model}

In this section, we further examine the linear system of equations (25) in some particular special cases of the model with a general penalty function. We start with the case where $n_{i}=1$ for all $i$, implying that $m=n$ and that the model involved is a (dependent) combination of exponential claim sizes. In this case the linear system (25) becomes (with $j=1$ )

$$
\sum_{k=1}^{n} \frac{C_{k, \delta}}{\beta_{i}-R_{k, \delta}}=\frac{E\left[w\left(E_{i, 1}\right)\right]}{\beta_{i}}, \quad i=1,2, \ldots, n,
$$

which is of Cauchy type. To solve for $C_{k, \delta}$ in (52), define

$$
Q^{*}(z)=\left\{\prod_{j=1}^{n}\left(z-R_{j, \delta}\right)\right\} \sum_{k=1}^{n} \frac{C_{k, \delta}}{z-R_{k, \delta}}=\sum_{k=1}^{n} C_{k, \delta}\left\{\prod_{j=1, j \neq k}^{n}\left(z-R_{j, \delta}\right)\right\} .
$$

For $i=1,2, \ldots, n,(52)$ implies that

$$
Q^{*}\left(\beta_{i}\right)=\frac{E\left[w\left(E_{i, 1}\right)\right]}{\beta_{i}}\left\{\prod_{j=1}^{n}\left(\beta_{i}-R_{j, \delta}\right)\right\}=E\left[w\left(E_{i, 1}\right)\right] \frac{\beta_{i}-R_{i, \delta}}{\beta_{i}} \prod_{j=1, j \neq i}^{n}\left(\beta_{i}-R_{j, \delta}\right) .
$$

As (53) is a polynomial of degree $n-1$ or less, the Lagrange interpolating polynomial argument yields

$$
Q^{*}(z)=\sum_{i=1}^{n} Q^{*}\left(\beta_{i}\right) \prod_{j=1, j \neq i}^{n}\left(\frac{\beta_{j}-z}{\beta_{j}-\beta_{i}}\right)
$$

i.e.,

$$
Q^{*}(z)=\sum_{i=1}^{n} E\left[w\left(E_{i, 1}\right)\right] \frac{\beta_{i}-R_{i, \delta}}{\beta_{i}} \prod_{j=1, j \neq i}^{n}\left\{\frac{\left(\beta_{j}-z\right)\left(\beta_{i}-R_{j, \delta}\right)}{\beta_{j}-\beta_{i}}\right\} .
$$

Substitution of $z=R_{k, \delta}$ into (53) and (54), and solving for $C_{k, \delta}$, yields

$$
C_{k, \delta}=\frac{\sum_{i=1}^{n} E\left[w\left(E_{i, 1}\right)\right] \frac{\beta_{i}-R_{i, \delta}}{\beta_{i}} \prod_{j=1, j \neq i}^{n}\left\{\frac{\left(\beta_{j}-R_{k, \delta}\right)\left(\beta_{i}-R_{j, \delta}\right)}{\beta_{j}-\beta_{i}}\right\}}{\prod_{j=1, j \neq k}^{n}\left(R_{k, \delta}-R_{j, \delta}\right)} .
$$


This result is consistent with Albrecher et al. (2010).

Turning now to the ladder height distribution (14) when $n_{i}=1$, one has $\widetilde{b}_{\delta}(s)=\sum_{i=1}^{n} \xi_{\delta, i 1}\left\{\beta_{i} /\left(\beta_{i}+s\right)\right\}$. Equating this to (38) yields

$$
\sum_{i=1}^{n} \beta_{i} \xi_{\delta, i 1}\left\{\prod_{j=1, j \neq i}^{n}\left(\beta_{j}+s\right)\right\}=\frac{1}{\phi_{\delta}}\left(\left\{\prod_{j=1}^{n}\left(s+\beta_{j}\right)\right\}-\left\{\prod_{j=1}^{n}\left(s+R_{j, \delta}\right)\right\}\right) .
$$

Substitution of $s=-\beta_{i}$ and solving for $\xi_{\delta, i 1}$ yields, for $i=1,2, \ldots, n$,

$$
\xi_{\delta, i 1}=\frac{\prod_{j=1}^{n}\left(\beta_{i}-R_{j, \delta}\right)}{\phi_{\delta} \beta_{i}\left\{\prod_{j=1, j \neq i}^{n}\left(\beta_{i}-\beta_{j}\right)\right\}} .
$$

To conclude, we briefly consider the special case where the marginal distribution of the claim sizes is a finite mixture of Erlangs with the same scale parameter (i.e. $m=1$ ). Under this distributional assumption, (25) becomes

$$
\sum_{k=1}^{n} C_{k, \delta}\left(\frac{\beta_{1}}{\beta_{1}-R_{k, \delta}}\right)^{j}=E\left[w\left(E_{1, j}\right)\right]
$$

and thus, $\left\{C_{k, \delta}\right\}_{k=1}^{n}$ are the solutions of the matrix equation

$$
\mathbf{A C}=\mathbf{W}
$$

where $\mathbf{A}=\left\{a_{j, k}\right\}_{n \times n}$ with $a_{j, k}=\left(\rho_{k}\right)^{j}$ and $\rho_{k}=\beta_{1} /\left(\beta_{1}-R_{k, \delta}\right)$, $\mathbf{C}$ is a column vector with $\left\{C_{k, \delta}\right\}_{k=1, \ldots, n}$ and $\mathbf{W}$ is a column vector with $\left\{E\left[w\left(E_{1, j}\right)\right]\right\}_{j=1, \ldots, n}$. Note that $\mathbf{A}$ is a Vandermonde matrix and its inverse $\mathbf{A}^{-1}=\mathbf{B}=\left\{b_{i, j}\right\}_{n \times n}$ has element

$$
b_{i, j}=\frac{\sum_{\substack{1 \leq k_{1}<\ldots<k_{n-j} \leq n \\ k_{1}, \ldots, k_{n-j} \neq i}}(-1)^{j} \rho_{k_{1} \ldots \rho_{k_{n-j}}}}{\rho_{i} \prod_{k=1, k \neq i}^{n}\left(\rho_{k}-\rho_{i}\right)},
$$

(e.g., Exercise 40 of Knuth (1997, Section 1.2.3)). It immediately follows that

$$
C_{k, \delta}=\sum_{j=1}^{n} b_{k, j} E\left[w\left(E_{1, j}\right)\right]
$$

for $k=1, \ldots, n$.

\section{$5 \quad$ References}

- Albrecher, H., Gerber, H.U., and Yang, H. 2010. A direct approach to the discounted penalty function. North American Actuarial Journal 14(4): 420-434.

- Albrecher, H., and Teugels, J.L. 2006. Exponential behavior in the presence of dependence in risk theory. Journal of Applied Probability 43(1): 257-273. 
- Badescu, A.L., Cheung, E.C.K., and Landriault, D. 2009. Dependent risk models with bivariate phase-type distributions. Journal of Applied Probability 46(1): 113-131.

- Boudreault, M., Cossette, H., Landriault, D., and Marceau, E. 2006. On a risk model with dependence between interclaim arrivals and claim sizes. Scandinavian Actuarial Journal 5: 265-285.

- Chadjiconstantinidis, S. and Vrontos, S. 2012. On a renewal risk process with dependence under a Farlie-Gumbel-Morgenstern copula. Scandinavian Actuarial Journal, DOI:10.1080/03461238.2012.6637

- Cheung, E.C.K. 2011. A generalized penalty function in Sparre Andersen risk models with surplus-dependent premium. Insurance: Mathematics and Economics 48(3): 384-397.

- Cheung, E.C.K., Landriault, D., Willmot, G.E., and Woo, J.-K. 2010. Structural properties of Gerber-Shiu functions in dependent Sparre Andersen models. Insurance: Mathematics and Economics 46(1): 117-126.

- Cossette, H., Marceau, E., and Marri, F. 2008. On the compound Poisson risk model with dependence based on a generalized Farlie-Gumble-Morgenstern copula. Insurance: Mathematics and Economics 43(3): 444-455.

- Gerber, H.U., and Shiu, E.S.W. 1998. On the time value of ruin. North American Actuarial Journal 2(1): 48-78.

- Knuth, D.E. 1997. The Art of Computer Programming: Volume 1: Fundamental Algorithms. 3rd edition, Addison-Wesley.

- Landriault, D., and Willmot, G.E. 2008. On the Gerber-Shiu discounted penalty function in the Sparre Andersen model with an arbitrary interclaim time distribution. Insurance: Mathematics and Economics 42(2): 600-608.

- Li, S., and Garrido, J. 2004. On ruin for the Erlang(n) risk process. Insurance: Mathematics and Economics 34(3): 391-408.

- Lin, X.S., and Willmot, G.E. 1999. Analysis of a defective renewal equation arising in ruin theory. Insurance: Mathematics and Economics 25(1): 63-84.

- Lin, X.S., and Willmot, G.E. 2000. The moments of the time of ruin, the surplus before ruin, and the deficit at ruin. Insurance: Mathematics and Economics 27(1): 19-44.

- Nelsen, R.B. 1994. A Characterization of Farlie-Gumbel-Morgenstern Distributions via Spearman's Rho and Chi-Square Divergence. Sankhyā: The Indian Journal of Statistics, Series A, 56(3): 476-479.

- Nelsen, R.B. 2006. An Introduction to Copulas. Springer Series in Statistics, 2nd edition, Springer-Verlag: New York.

- Pitts, S.M., and Politis, K. 2007. The joint density of the surplus before and after ruin in the Sparre Andersen model. Journal of Applied Probability 44(3): 695-712.

- Sancetta, A., and Satchell, S. 2004. The Bernstein copula and its applications to modeling and approximations of multivariate distributions. Econometric Theory 20: 535-562. 
- Tang, Q., and Wei, L. 2010. Asymptotic aspects of the Gerber-Shiu function in the renewal risk model using Wiener-Hopf factorization and convolution equivalence. Insurance: Mathematics and Economics 46(1): 19-31.

- Willmot, G.E., and Lin, X.S. 2000. Lundberg Approximations for Compound Distributions with Insurance Applications. Lecture Notes in Statistics 156. Springer-Verlag: New York.

- Willmot, G.E. 2007. On the discounted penalty function in the renewal risk model with general interclaim times. Insurance: Mathematics and Economics 41: 17-31.

- Willmot, G.E., and Woo, J.-K. 2012. On the analysis of a general class of dependent risk processes. Insurance: Mathematics and Economics 51(1): 134-141.

- Zhang, Z., Yang, H., and Yang, H. 2011. On a Sparre Andersen risk model with time- dependent claim sizes and jump-diffusion perturbation. Methodology and Computing in Applied Probability 1-23. 